\title{
Role of tissue transglutaminase in the pathogenesis of diabetic cardiomyopathy and the intervention effect of rutin
}

\author{
HAI-CHENG GAO ${ }^{1 *}, \mathrm{KUN}_{\text {ZHU }}{ }^{*}$, HAI-MEI GAO ${ }^{3}$, CHUN-SHENG MIAO $^{1}$, \\ LE-NING ZHANG ${ }^{4}$, WEI LIU ${ }^{2}$ and HUA XIN ${ }^{4 *}$

\begin{abstract}
${ }^{1}$ Department of Clinical Pharmacy and Pharmaceutical Management, Jilin University, Changchun, Jilin 130021; ${ }^{2}$ Department of Endocrinology, The 208th Hospital of PLA, Changchun, Jilin 130000; ${ }^{3}$ Patient's Information Recording Room, The Second Hospital of Jilin University, Changchun, Jilin 130041; ${ }^{4}$ Department of Chest Surgery,

The China-Japan Union Hospital of Jilin University, Changchun, Jilin 130000, P.R. China
\end{abstract}

Received April 17, 2014; Accepted December 1, 2014

DOI: $10.3892 /$ etm.2015.2223

\begin{abstract}
The aim of this study was to investigate the role of tissue transglutaminase (tTG) in the pathogenesis of diabetic cardiomyopathy (DCM) and the intervention effect of rutin. DCM was induced in rats by the injection of streptozotocin (STZ; $25 \mathrm{mg} / \mathrm{kg}$ ). After a preliminary examination, the rats were randomly divided into four groups: Control $(n=8)$, STZ-induced $\operatorname{DCM}(n=8), S T Z$ + positive drug (captopril; $n=6)$ and STZ + rutin $(n=8)$ groups. The DCM model was evaluated using blood sugar values, serum enzyme levels, hematoxylin and eosin staining and Masson's staining, ex vivo. The protein and mRNA expression of tTG was assessed with immunohistochemistry, western blotting and reverse transcription-quantitative polymerase chain reaction (RT-qPCR). The rat model of DCM was successfully established by STZ administration, and the expression levels of tTG were significantly increased in the DCM model. Following the injection of captopril or rutin, the blood sugar values, collagen content and expression levels of tTG were gradually reduced and serum enzyme levels were increased, as compared with those in the STZ-induced DCM group. In conclusion, tTG plays an important role in STZ-induced DCM. In addition, rutin may inhibit the expression of $\mathrm{tTG}$ and regulate myocardial injury in STZ-induced DCM.
\end{abstract}

\section{Introduction}

Diabetes mellitus (DM) can cause many complications, including heart disease, kidney failure (1) and diabetic cardio-

Correspondence to: Professor Hua Xin, Department of Chest Surgery, The China-Japan Union Hospital of Jilin University, 1266 Fujin Road, Changchun, Jilin 130000, P.R. China

E-mail: gaohc@jlu.edu.cn

*Contributed equally

Key words: diabetic, diabetic cardiomyopathy, tissue transglutaminase, rutin myopathy (DCM) (2). DCM can lead to the heart being unable to circulate blood through the body effectively (heart failure), with accumulation of fluid in the lungs or legs (3). At present, heart failure in people with diabetes primarily results from coronary artery disease, and DCM is only considered to exist if no coronary artery disease is present to explain the heart muscle disorder (4). Numerous factors have been identified to be involved in the pathogenesis of DM and cardiomyopathy, including MMP-2 and ICAM-1 (5).

Tissue transglutaminase (tTG), a member of the transglutaminase family (6), is $\mathrm{Ca}^{2+}$ dependent (7) and is reported to play an important role in myocardial injury (8). tTG is present in the intracellular and extracellular spaces of various types of tissues and in various organs, including the heart and liver (8). In the extracellular space, tTG is associated with cell adhesion, extracellular matrix stabilization, wound healing, receptor signaling, cellular proliferation and cellular motility (9). It is unclear whether tTG plays an important role in STZ-induced DCM, and if it does, whether drugs can be used to inhibit the expression of tTG and regulate the occurrence and development of DCM. In the past decade, numerous drugs with potential for the treatment of DCM have been identified, for example sulforaphane (10), but the treatment effect requires improvement. Therefore, the development of drugs to treat DCM is urgently necessary. Rutin has the apparent ability to strengthen blood vessels, and can reduce and delay the development of diabetes (11). However, whether rutin is able to prevent and treat DCM is unclear.

The present study investigated blood sugar values, cardiac enzyme levels, the expression of tTG and collagen I (Col-I) in the heart and the interventional effect of rutin in a rat model of DCM.

\section{Materials and methods}

Materials. Streptomycin avidin peroxidase immunohistochemistry (SP-IHC) kits for tTG and Col-I were purchased from Zhongshan Jinqiao Biotechnology Co., Ltd. (Beijing, China). Captopril tablets were from Zhengzhou Ruikang Pharmaceutical Co., Ltd. (Zhengzhou, China), rutin was from Xi'an Tianxingjian Industry Co., Ltd. (Xi'an, China) and 
streptozotocin (STZ) was from Sigma-Aldrich (St. Louis, MO, USA). All other chemicals were obtained from commercial suppliers in China.

Animals. A total of 30 male Wistar rats (SCXKJ2007-0003; 180-220 g) were purchased from Jilin University Laboratory Animal Center (Changchun, China) and were randomized into four groups: control $(n=8)$, STZ-induced DCM $(n=8)$, STZ + positive drug (captopril tablets; $n=6$ ) and STZ + rutin $(\mathrm{n}=8)$ groups. The rat model of DCM was established by the peritoneal injection of STZ $(25 \mathrm{mg} / \mathrm{kg})$. Captopril and rutin were administered at doses of 5 and $40 \mathrm{mg} / \mathrm{kg} / \mathrm{day}$, respectively, by gastric lavage. The treatment began 21 days after the injection of STZ and was administered for 28 days. At the end of the experiments (day 49 after STZ injection), the rats were sacrificed and their hearts were removed. A portion of the heart was fixed in $10 \%$ phosphate-buffered formalin, frozen in liquid nitrogen and stored at $-80^{\circ} \mathrm{C}$ for extraction. The study was approved by the ethics committee of the College of Pharmacy of Jilin University (Changchun, China).

Blood sugar values. The Wistar rats were reared for one month by feeding with a high-sugar diet. The blood sugar levels of the rats were then measured using test strips (Sannuo Bio-Sensing Technology Co., Ltd., Changsha, China).

Analysis of serum enzymes. Serum aspartate aminotransferase (AST), lactic dehydrogenase (LDH), creatine kinase (CK) and creatine kinase isoenzyme MB (CK-MB) activities were measured using the MD-100 Multifunctional Automatic Biochemistry Analyzer (Sanhe Medical Equipment Co., Ltd., Dandong, China) according to the manufacturer's instructions.

Hematoxylin and eosin $(H \& E)$ and Masson's staining. Renal histology was assessed by light microscopy with H\&E staining and Masson's trichrome staining. Heart tissues were harvested and fixed in neutral buffered $10 \%$ or $4 \%$ paraformaldehyde. After paraffin embedding, heart tissue sections were prepared $(0.5 \mu \mathrm{m})$ and then subjected to H\&E and Masson's staining for morphological observation using a PM-10AO automatic photomicrographic device (Olympus Co., Ltd., Tokyo, China). Ten high-power microscopic fields were randomly selected and the percentage of Masson staining was determined from the average gray value of five rats in each group.

Immunohistochemical staining. The paraffin-embedded tissue sections $(0.5 \mu \mathrm{m})$ were deparaffinized with xylene and rehydrated with graded washes of ethanol to phosphate-buffered saline (PBS). Staining was conducted using SP-IHC kits. In this process, each slice was treated with $30 \mu \mathrm{l} 3 \% \mathrm{H}_{2} \mathrm{O}_{2}$ (reagent A), incubated at room temperature for $20 \mathrm{~min}$ and washed twice with PBS. Then, $30 \mu 1$ goat serum (reagent B) was added, followed by incubation at room temperature for $20 \mathrm{~min}$ and two washes with PBS. Each slice was incubated in $30 \mu \mathrm{l}$ primary antibody and placed in a wet box at $4^{\circ} \mathrm{C}$ overnight. The primary antibody was rabbit anti-rat tTG polyclonal antibody (1:200 dilution) or rabbit anti-rat Col-I monoclonal antibody, (1:150 dilution). After washing with PBS, the slices were incubated in $30 \mu 1$ biotinylated polyclonal secondary antibody (reagent $\mathrm{C}$ ) at room temperature for $30 \mathrm{~min}$, followed by washing with PBS. The diaminobenzidine (DAB) method was used for color development, followed by washing with tap water. Slices were restained with hematoxylin, incubated in ammonia, dehydrated with gradient ethanol, transparentized with xylene and finally sealed with neutral gum. The cells with brown particles in their cytoplasm and nucleus when observed under a light microscope were considered positive. The expression levels were determined as the average gray value of five rats in each group.

Western blotting. Heart tissues were homogenized in protein lysis buffer and $50 \mathrm{~g}$ proteins were separated on $10 \%$ sodium dodecyl sulfate (SDS) gels and electroblotted to polyvinylidine fluoride membranes. The membranes were blocked with skimmed milk powder solution for $2 \mathrm{~h}$. Blots were incubated using rabbit anti-rat tTG polyclonal antibody (Santa Cruz Biotechnology, Inc., Dallas, TX, USA) at $4^{\circ} \mathrm{C}$ overnight, followed by peroxidase-conjugated goat anti-rabbit IgG polyclonal antibody (dilution 1:2,000: Boster Bio-Engineering Co., Ltd, Wuhan, China) for $2 \mathrm{~h}$ at $37^{\circ} \mathrm{C}$. Color was developed with an enhanced chemiluminescence (ECL) reagent (Thermo Fisher Scientific Inc., Waltham, MA, USA) in a dark room. The gray scale values were analyzed using the Tanon-1000 Gel Image System (Tanon, Shanghai, China).

Reverse transcription-quantitative polymerase chain reaction $(R T-q P C R)$. Heart tissue $(100 \mathrm{mg})$ was ultrasonically homogenized with $1 \mathrm{ml}$ TRIzol, transferred into Eppendorf tubes and incubated at room temperature with $0.2 \mathrm{ml}$ chloroform for $5 \mathrm{~min}$. The samples were then shocked at room temperature $\left(15-30^{\circ} \mathrm{C}\right)$ for $15 \mathrm{sec}$, incubated for 2-3 min and centrifuged at $12,000 \mathrm{x} \mathrm{g}$ for $15 \mathrm{~min}\left(2-8^{\circ} \mathrm{C}\right)$. The supernatant was transferred into a new tube. To each tube was added $0.5 \mathrm{ml}$ isopropanol at room temperature, and after $10 \mathrm{~min}$ the tubes were centrifuged at $12,000 \mathrm{x}$ g for $10 \mathrm{~min}$. The supernatant was removed, $1 \mathrm{ml}$ $75 \%$ ethanol was added and the mixture was centrifuged at $7,500 \mathrm{xg}$ for $5 \mathrm{~min}$ to provide total RNA. A sample of the total RNA $(2 \mu \mathrm{l})$ was reversed transcribed to cDNA. In this process, the RNA was dissolved in $30 \mu 1$ diethylpyrocarbonate-treated water. First-strand DNA was synthesized using an RT reaction system $(20 \mu \mathrm{l})$ as follows: $10 \mu \mathrm{l}$ deionized water with no RNA enzyme, $2 \mu \mathrm{l}$ template RNA, $180 \mu \mathrm{l}$ Oligo(dT), $4 \mu 1$ 5X reaction buffer, $1 \mu 1$ RNase inhibitor (20 U/ $/$ l), $2 \mu \mathrm{l}$ dNTP mix (10 $\mathrm{mmol} / \mathrm{l}$ ) and $1 \mu \mathrm{l}$ SuperScript II RT (TransGen Biotech Co., Ltd., Beijing, China). The reaction conditions were as follows: $70^{\circ} \mathrm{C}$ for $5 \mathrm{~min}, 37^{\circ} \mathrm{C}$ for $5 \mathrm{~min}, 37^{\circ} \mathrm{C}$ for $60 \mathrm{~min}, 70^{\circ} \mathrm{C}$ for $10 \mathrm{~min}$ and then placed on ice for subsequent testing or preservation at $-80^{\circ} \mathrm{C}$. The primers for tTG were 5 '-AAGGGAAGT CTTCACCAGAGCCAA-3' (sense) and 5'-CGATGTGGG CAAACACGTCAAAGT-3' (anti-sense). PCR was conducted using Taq DNA polymerase (TransGen Biotech Co., Ltd.) and the following amplification protocol: 1 cycle at $95^{\circ} \mathrm{C}$ for $5 \mathrm{~min}$ followed by 30 cycles consisting of $30 \mathrm{sec}$ at $95^{\circ} \mathrm{C}, 45 \mathrm{sec}$ at $55^{\circ} \mathrm{C}$ and $1 \mathrm{~min}$ at $72^{\circ} \mathrm{C}$, and a final $10 \mathrm{~min}$ extension at $72^{\circ} \mathrm{C}$ in an automated thermal cycler (PCR Express; Thermo Fisher Scientific).

Statistical analyses. All statistical analyses were performed using SPSS version 15.0 for Windows (SPSS Inc., Chicago, IL, USA). Comparisons between multiple groups were 
Table I. Blood sugar levels in each group ( $\mathrm{mmol} / \mathrm{l})$.

\begin{tabular}{lcccc}
\hline Groups & N & 35 days & 42 days & 49 days \\
\hline Control & 8 & $6.20 \pm 0.55$ & $5.50 \pm 0.24$ & $6.44 \pm 0.24$ \\
STZ-induced DCM & 8 & $18.10 \pm 2.41^{\mathrm{a}}$ & $18.00 \pm 2.81^{\mathrm{a}}$ & $16.50 \pm 3.50^{\mathrm{a}}$ \\
STZ + positive drug & 6 & $15.01 \pm 6.80^{\mathrm{b}}$ & $13.90 \pm 3.18^{\mathrm{b}}$ & $14.20 \pm 2.63^{\mathrm{b}}$ \\
STZ + rutin & 8 & $18.36 \pm 2.46$ & $14.21 \pm 2.63^{\mathrm{b}}$ & $15.85 \pm 3.05$
\end{tabular}

Values are mean \pm standard deviation. STZ, streptozotocin; DCM, diabetic cardiomyopathy; positive drug, captopril. ${ }^{\mathrm{a}} \mathrm{P}<0.05$ vs. control, ${ }^{\mathrm{b}} \mathrm{P}<0.05$ vs. STZ-induced DCM.

Table II. Serum enzyme values in each group $(\mu / 1)$.

\begin{tabular}{llllll}
\hline Groups & N & AST & LDH & CK & CK-MB \\
\hline Control & 5 & $100.6 \pm 6.7$ & $1834.4 \pm 429.9$ & $1002.2 \pm 120.0$ & $999.9 \pm 128.0$ \\
STZ-induced DCM & 5 & $124.8 \pm 17.4^{\mathrm{a}}$ & $2255.4 \pm 69.1^{\mathrm{a}}$ & $1341.6 \pm 124.9^{\mathrm{a}}$ & $1339.3 \pm 120.3^{\mathrm{a}}$ \\
STZ + positive drug & 5 & $102.8 \pm 2.0^{\mathrm{b}}$ & $1999.4 \pm 135.6^{\mathrm{b}}$ & $1198.6 \pm 65.3^{\mathrm{b}}$ & $1023.5 \pm 90.8^{\mathrm{b}}$ \\
STZ + rutin & 5 & $113.0 \pm 4.4^{\mathrm{b}}$ & $2199.2 \pm 93.7$ & $1419.0 \pm 120.5$ & $1333.8 \pm 112.9$ \\
\hline
\end{tabular}

Values are mean \pm standard deviation. STZ, streptozotocin; DCM, diabetic cardiomyopathy; positive drug, captopril; AST, aspartate aminotransferase; $\mathrm{LDH}$, lactate dehydrogenase; $\mathrm{CK}$, creatine kinase; $\mathrm{CK}-\mathrm{MB}$, creatine kinase isenozyme $\mathrm{MB}$. ${ }^{\mathrm{P}}<0.05$ vs. control, ${ }^{\text {b }} \mathrm{P}<0.05$ vs. STZ-induced DCM.
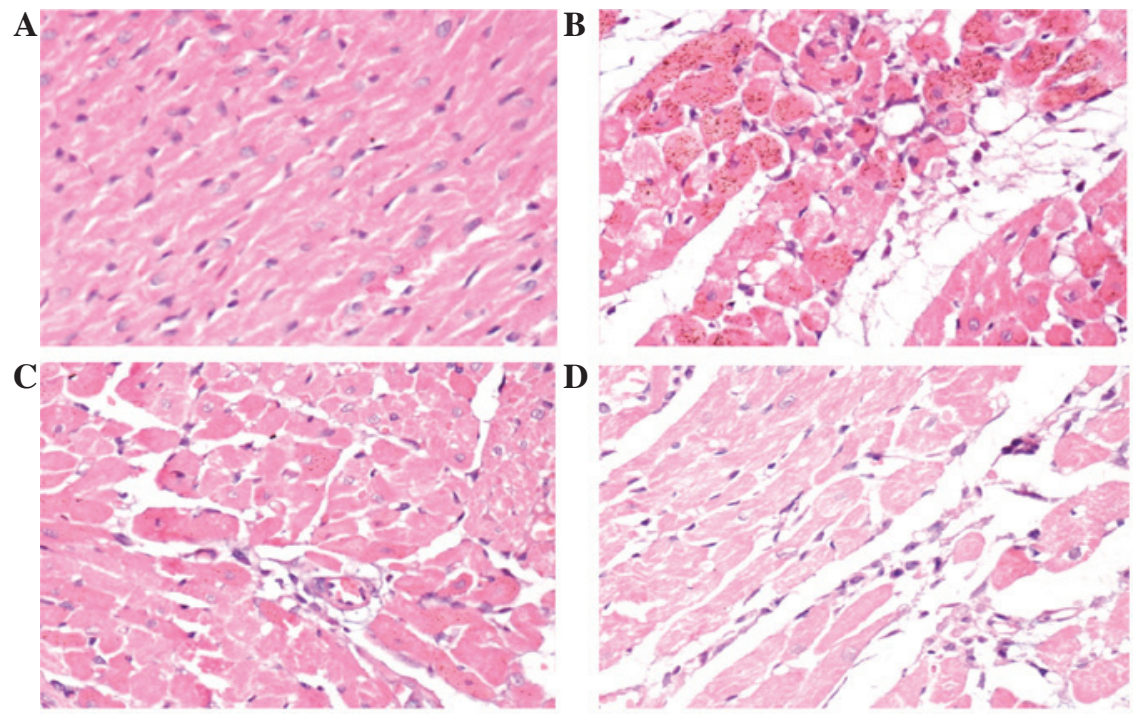

Figure 1. Histopathological images. Tissue samples were preserved in $10 \%$ neutral buffered formalin, paraffin embedded, processed to slides, and stained with hematoxylin and eosin. Representative histopathological images are shown. (A) Normal, (B) model (STZ-induced DCM), (C) STZ + positive drug (captopril) and (D) STZ + rutin. Magnification, x400. STZ, streptozotocin; DCM, diabetic cardiomyopathy.

performed by one-way analysis of variance (ANOVA). $\mathrm{P}<0.05$ was considered to indicate a statistically significant difference.

\section{Results}

Blood sugar values. Blood sugar measurements were obtained from the 30 rats. As shown in Table I, the blood sugar levels in the model group were higher than those in the control group $(\mathrm{P}<0.05)$. This indicated that the diabetic rat model was successfully constructed. The blood sugar levels in the STZ + positive drug and STZ + rutin groups were lower than those in the model group on days 42 and 49 $(\mathrm{P}<0.05)$. These results indicate that rutin may have a role in blood sugar reduction.

Serum enzyme values. The activity levels of AST, LDH, CK and $\mathrm{CK}-\mathrm{MB}$ in the model group were lower than those in the control group $(\mathrm{P}<0.05)$. These results showed that STZ might induce myocardial injury. The activity levels of AST, LDH, 

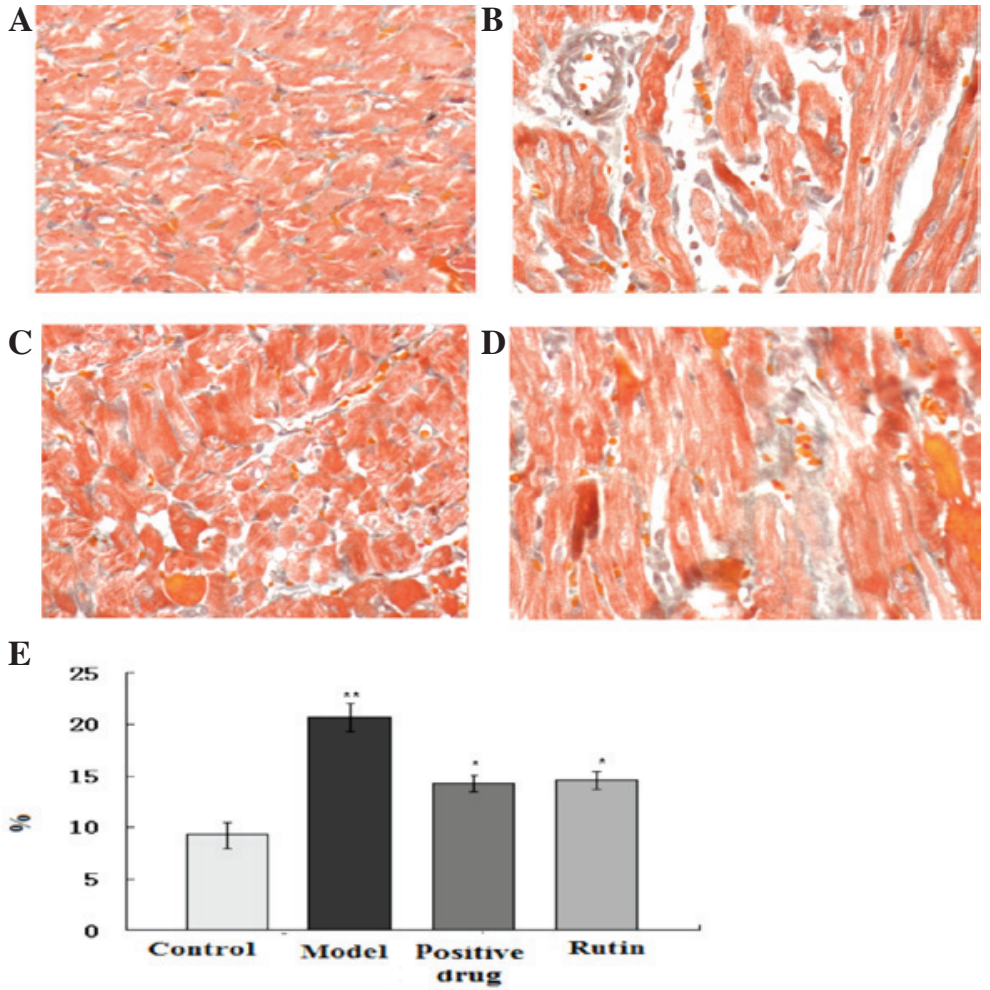

Figure 2. Masson trichrome staining. (A) Normal, (B) model (STZ-induced DCM), (C) positive drug (STZ + captopril) and (D) rutin (STZ + rutin) (E) Percentage of Masson trichrome staining in the various groups. STZ, streptozotocin; DCM, diabetic cardiomyopathy. Magnification, $\mathrm{x} 1,000$. " $\mathrm{P}<0.05 \mathrm{vs}$. model; ${ }^{* *} \mathrm{P}<0.01$ vs. control.

A

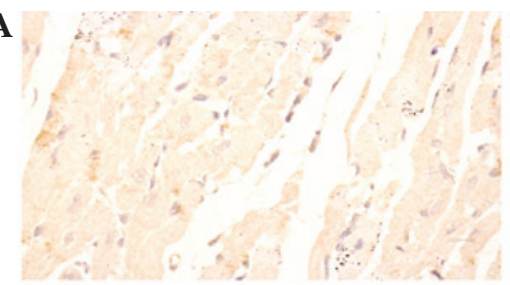

C

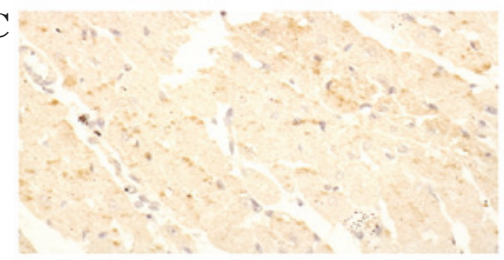

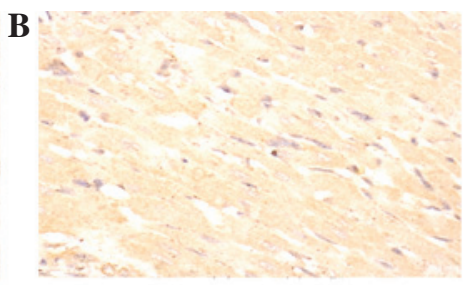

D

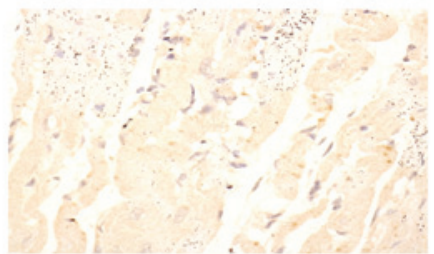

$\mathbf{E}$

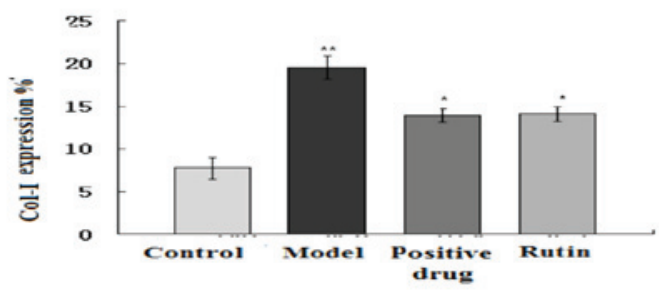

Figure 3. Immunohistochemistry of collagen I (Col-I). (A) Normal, (B) model (STZ-induced DCM), (C) positive drug (STZ + captopril) and (D) rutin (STZ + rutin). (E) Percentage of Col-I staining in the various groups. STZ, streptozotocin; DCM, diabetic cardiomyopathy. Magnification, $\mathrm{x} 1,000$. " $\mathrm{P}<0.05 \mathrm{vs.}$ model; ${ }^{* *} \mathrm{P}<0.01$ vs. control.

$\mathrm{CK}$ and CK-MB in the STZ + positive drug and STZ + rutin groups were higher than those in the model group $(\mathrm{P}<0.05)$. These results indicate that rutin may have a role in increasing the activity levels of AST, LDH, CK and CK-MB (Table II).
$H \& E$ staining. A morphological assessment, as the gold standard for disease diagnosis, is the most reliable method for diagnosing organ injury. Examination of the rat heart tissue slices revealed that there was evident denaturation and edema in the apex cordis 

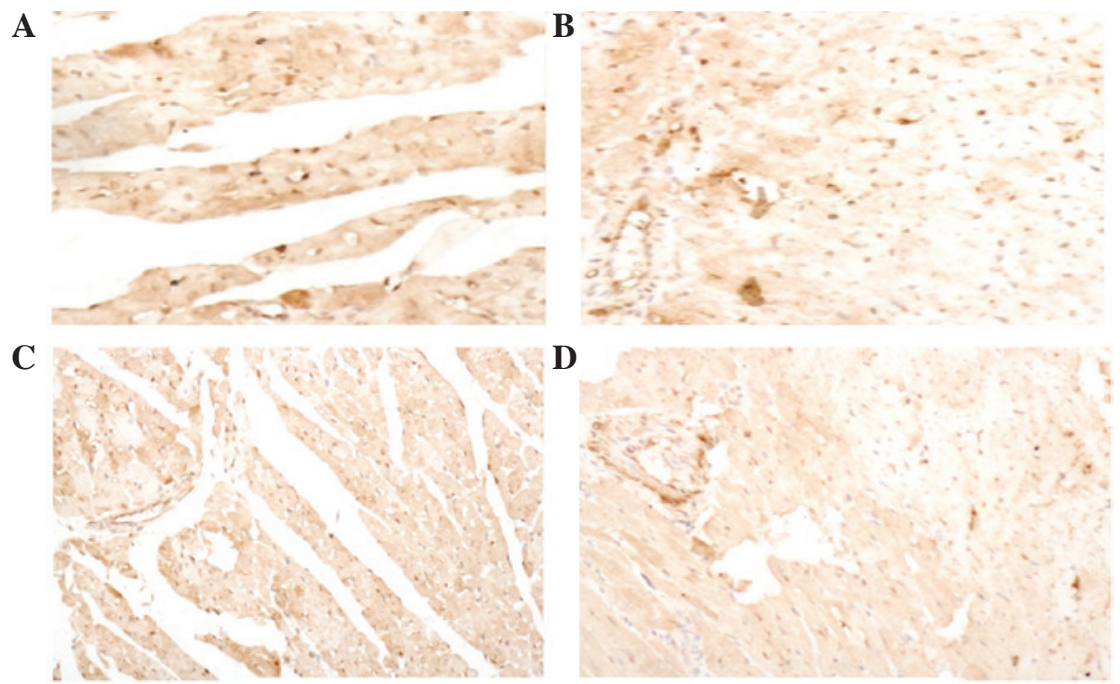

$\mathbf{E}$

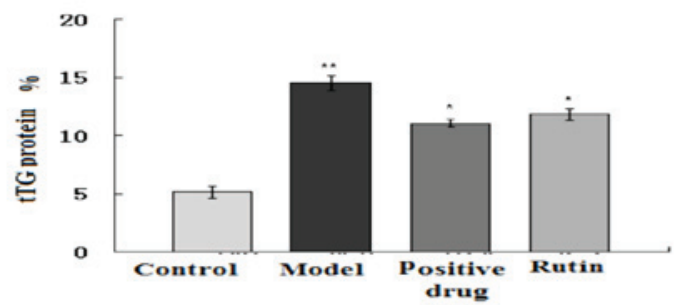

Figure 4. Tissue transglutaminase (tTG) immunohistochemical staining. (A) Normal, (B) model (STZ-induced DCM), (C) positive drug (STZ + captopril) and (D) rutin (STZ + rutin). (E) Percentage of tTG staining in the various groups. STZ, streptozotocin; DCM, diabetic cardiomyopathy. Magnification, x1,000. ${ }^{*} \mathrm{P}<0.05$ vs. model; ${ }^{* * *} \mathrm{P}<0.01$ vs. control.

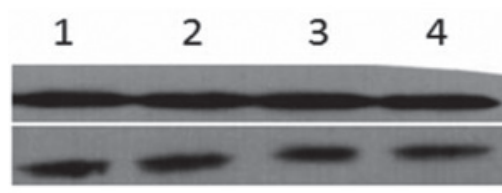

38KD GAPDH

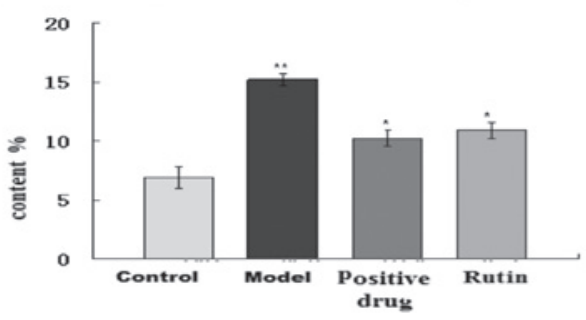

Figure 5. Results of western blotting. Representative western blots and tTG expression levels in the various groups. Lane 1, normal (control) group; lane 2, model (STZ-induced DCM); lane 3, positive drug (STZ + captopril); lane 4, rutin (STZ + rutin). STZ, streptozotocin; DCM, diabetic cardiomyopathy; tTG, tissue transglutaminase. ${ }^{*} \mathrm{P}<0.05$ vs. model; ${ }^{* *} \mathrm{P}<0.01$ vs. control.

and endocardium following the injection of STZ. Myocardial tissues appeared necrotic in the endocardium and interstitium. These results suggest that heart injury was successfully generated. Following the injection of captopril or rutin, there was a certain improvement in the degree of myocardial injury. These results indicated that rutin may have a protective effect against STZ-induced myocardial injury (Fig. 1).

Masson's staining. Experimental results demonstrated that the collagen content in the model group was higher than that in the control group following the injection of STZ $(\mathrm{P}<0.05)$.

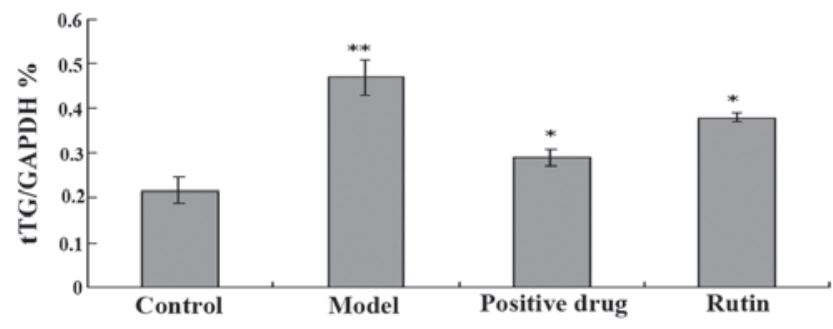

Figure 6. Results of RT-qPCR. mRNA expression of tTg in the rat myocardium of the control, model (STZ-induced DCM), positive drug (STZ + captopril) and rutin (STZ + rutin) groups. RT-qPCR, reverse transcription-quantitative polymerase chain reaction. tTG, tissue transglutaminase; STZ, streptozotocin; DCM, diabetic cardiomyopathy. ${ }^{*} \mathrm{P}<0.05$ vs. model; ${ }^{* *} \mathrm{P}<0.01$, vs. control.

The collagen contents in the groups treated with captopril or rutin were lower than that in the model group $(\mathrm{P}<0.05)$. These results indicate that treatment with rutin reduces collagen content (Fig. 2).

Col-I staining. As shown in Fig. 3, the expression level of Col-I in the model group was higher than that in the control group $(\mathrm{P}<0.05)$. After treatment with captopril or rutin, the expression level of Col-I was lower than that in the model group $(\mathrm{P}<0.05)$.

tTG staining. In the control group, tTG immunoreactivity was weakly detected in the myocardial interstitial region (Fig. 4A). In this group, tTG immunoreactivity was observed in blood vessel-like structures. The expression level of tTG increased markedly in the model group as compared with 
that in the control group $(\mathrm{P}<0.05)$. The expression levels of tTG in the groups treated with captopril or rutin were observed to be lower than that in the model group $(\mathrm{P}<0.05)$. These results suggest that treatment with rutin inhibits the expression of tTG.

Expression of $t$ T $G$ as determined by western blotting. Western blotting was used to investigate the expression of tTG. The results were analyzed semi-quantitatively according to the grayscale value. As shown in Fig. 5, the expression level of tTG in the model group was higher than that in the model group $(\mathrm{P}<0.05)$. The expression levels of tTG protein in the rats treated with captopril or rutin were lower than that in the model group $(\mathrm{P}<0.05)$.

$R T-q P C R$ results. RT-qPCR was used to investigate the mRNA expression of tTG. As shown in Fig. 6, the mRNA expression level of tTG in the model group was higher than that in the control group $(\mathrm{P}<0.05)$. The expression levels of tTG mRNA in the rats treated with captopril or rutin were lower than that in the model group $(\mathrm{P}<0.05)$.

\section{Discussion}

In patients with DM, the cardiac structure and function may be altered in the absence of changes in blood pressure and coronary artery disease, a condition known as DCM. DCM is ventricular dysfunction that occurs independently of coronary artery disease and hypertension. In DCM, the diastolic dysfunction becomes more apparent in the presence of hypertension or myocardial ischemia (12). tTG is the autoantigen in coeliac disease, an illness in which dietary gluten causes a pathological immune response resulting in inflammation of the small intestine and subsequent villous atrophy (13). In the present study, the changes of tTG in the myocardial interstitial region were observed following STZ-induced myocardial injury. The results indicated that the expression levels of tTG protein and mRNA were increased in the myocardial interstitial region following injury. A previous study reported that the expression levels of tTG protein and mRNA in normal human organs were lower than those in injured organs (14). Furthermore, other researchers have suggested a role for tTG in heart mechanisms (15). In a previous study, significant increases of tTG protein and mRNA transcription levels were observed in ischemic Wistar rats at five months following STZ-induction compared with the levels in the sham-injured heart (16). These results indicate that myocardial injury is induced by the injection of STZ. Rutin has strong antioxidant properties, and the ability to chelate metal ions, such as iron, and reduce the Fenton reaction (13). Rutin is important due to its effect in strengthening capillaries. A previous animal study showed that it has preventive and healing effects (17). This indicates the possibility that rutin may play an important role in DCM. In the present study, the expression levels of tTG protein and mRNA, blood sugar levels, serum enzyme levels and collagen content were reduced gradually in the DCM model rats following the injection of rutin. These results indicate that rutin may have a preventive effect for DCM.
In conclusion, tTG may play an important role in STZ-induced DCM. In addition, rutin may inhibit the expression of tTG protein and gene, and regulate blood sugar values and serum enzymes levels. However, the results require verification in further studies.

\section{Acknowledgements}

This study was supported by the Science and Technology Department of Jilin Province, China (nos. 201101067 and 3D511B873432) and the Basic Research Foundation of Jilin University (no. 450060481127).

\section{References}

1. Pelle AJ, Loerbroks A, Widdershoven JW and Denollet J: Heart failure and comorbid diabetes mellitus or chronic obstructive pulmonary disease: effects on mood in outpatients. Int $\mathrm{J}$ Cardiol 154: 216-217, 2012.

2. Li B, Zheng Z, Wei Y, et al: Therapeutic effects of neuregulin-1 in diabetic cardiomyopathy rats. Cardiovasc Diabetol 10: 69, 2011.

3. Asghar O, Al-Sunni A, Khavandi K, et al: Diabetic cardiomyopathy. Clin Sci (Lond) 116: 741-760, 2009.

4. Avogaro A, Vigili de Kreutzenberg S, Negut C, Tiengo A and Scognamiglio R: Diabetic cardiomyopathy: a metabolic perspective. Am J Cardiol 93: 13A-16A, 2004.

5. Lee SW, Song KE, Shin DS, et al: Alterations in peripheral blood levels of TIMP-1, MMP-2, and MMP-9 in patients with type-2 diabetes. Diabetes Res Clin Pract 69:175-9, 2005.

6. Tucholski J and Johnson GV: Tissue transglutaminase differentially modulates apoptosis in a stimuli-dependent manner. J Neurochem 81: 780-791, 2002.

7. Barsigian C, Stern AM and Martinez J: Tissue (type II) transglutaminase covalently incorporates itself, fibrinogen, or fibronectin into high molecular weight complexes on the extracellular surface of isolated hepatocytes. Use of 2-[(2-oxopropyl)thio] imidazolium derivatives as cellular transglutaminase inactivators. J Biol Chem 266: 22501-22509, 1991.

8. Song H, Kim BK, Chang W, et al: Tissue transglutaminase 2 promotes apoptosis of rat neonatal cardiomyocytes under oxidative stress. J Recept Signal Transduct Res 31: 66-74, 2011.

9. Lauzier A, Charbonneau M, Paquette M, Harper K and Dubois CM: Transglutaminase 2 cross-linking activity is linked to invadopodia formation and cartilage breakdown in arthritis. Arthritis Res Ther 14: R159, 2012.

10. Zhang Z, Wang S, Zhou S, et al: Sulforaphane prevents the development of cardiomyopathy in type 2 diabetic mice probably by reversing oxidative stress-induced inhibition of LKB1/AMPK pathway. J Mol Cell Cardiol: Sep 28, 2014 (Epub ahead of print).

11. Kappel VD, Cazarolli LH, Pereira DF, et al: Involvement of GLUT-4 in the stimulatory effect of rutin on glucose uptake in rat soleus muscle. J Pharm Pharmacol 65: 1179-1186, 2013.

12. Boudina S and Abel ED: Diabetic cardiomyopathy revisited. Circulation 115: 3213-3223, 2007.

13. Mäki M: Tissue transglutaminase as the autoantigen of coeliac disease. Gut 41: 565-566, 1997.

14. Liao CW, Fan CK, Kao TC, et al: Brain injury-associated biomarkers of TGF-beta1, S100B, GFAP, NF-L, tTG, AbetaPP, and tau were concomitantly enhanced and the UPS was impaired during acute brain injury caused by Toxocara canis in mice. BMC Infect Dis 8: 84, 2008.

15. Petrak J, Pospisilova J, Sedinova M, et al: Proteomic and transcriptomic analysis of heart failure due to volume overload in a rat aorto-caval fistula model provides support for new potential therapeutic targets - monoamine oxidase A and transglutaminase 2. Proteome Sci 9: 69, 2011.

16. Mirza A, Liu SL, Frizell E, et al: A role for tissue transglutaminase in hepatic injury and fibrogenesis, and its regulation by NF-kappaB. Am J Physiol 272: G281-G288, 1997.

17. Hao HH, Shao ZM, Tang DQ, et al: Preventive effects of rutin on the development of experimental diabetic nephropathy in rats. Life Sci 91: 959-967, 2012. 